\title{
Light-by-light scattering in ultraperipheral heavy-ion collisions at low diphoton masses
}

\author{
Mariola Kłusek-Gawenda, ${ }^{1, *}$ Ronan McNulty, ${ }^{2, \dagger}$ Rainer Schicker, ${ }^{3, \ddagger}$ and Antoni Szczurek ${ }^{1, \S}$ \\ ${ }^{1}$ Institute of Nuclear Physics Polish Academy of Sciences, \\ Radzikowskiego 152, PL-31-342 Kraków, Poland \\ ${ }^{2}$ School of Physics, University College Dublin, Dublin 4, Ireland \\ ${ }^{3}$ Physikalisches Institut, Ruprecht-Karls-Universität Heidelberg, Heidelberg D69120, Germany
}

(Received 5 April 2019; published 30 May 2019)

\begin{abstract}
We present a study of photon-photon scattering in the mass range $W_{\gamma \gamma}<5 \mathrm{GeV}$. We extend earlier calculations of this cross section for $W_{\gamma \gamma}>5 \mathrm{GeV}$ into the low mass range where photoproduction of the pseudoscalar resonances $\eta, \eta^{\prime}(958)$ contributes to two-photon final states. We present the elementary photon-photon cross section as a function of diphoton mass $M_{\gamma \gamma}$ arising from lepton and quark loop diagrams, and the visible cross section obtained with the gamma-gamma decay branching fractions of the resonances $\eta, \eta^{\prime}(958), \eta_{c}(1 S), \eta_{c}(2 S), \chi_{c 0}(1 P)$. We derive the corresponding cross sections in ultraperipheral $\mathrm{Pb}-\mathrm{Pb}$ collisions at $\sqrt{s_{N N}}=5.02 \mathrm{TeV}$ by folding the elementary cross section with the heavyion photon fluxes. We consider the dominating background of the two photon final state which arises from gamma decays of photoproduced $\pi^{0}$-pairs. Such $\pi^{0}$-pairs contribute to the background when only two of the four decay photons are within the experimental acceptance, while the other two photons escape undetected. We reduce this background by applying cuts on asymmetries of transverse momenta of the two photons and indicate how the background can be further suppressed using a multivariate sideband analysis. We present the cross section for the signal and the background at midrapidity $|\eta|<0.9$, and in the forward rapidity range $2.0<\eta<4.5$.
\end{abstract}

DOI: 10.1103/PhysRevD.99.093013

\section{INTRODUCTION}

The properties of light have at all times fascinated physicists. The development of instruments for measuring light, and the design of experiments using light, have resulted in fundamental contributions to our current knowledge of modern physics. Our present understanding of the behavior of light at the classical level is conveniently expressed by Maxwell's equations. These equations are linear in the electric currents as sources, and in the resulting electric and magnetic fields $\mathbf{E}$ and $\mathbf{B}$. At the classical level, two electromagnetic waves in vacuum will superimpose, and will pass through each other without scattering. With the emergence of quantum mechanics, first attempts to

\footnotetext{
*Mariola.Klusek@ifj.edu.pl

†Ronan.Mcnulty@cern.ch

schicker@physi.uni-heidelberg.de

§Antoni.Szczurek@ifj.edu.pl;

Also at Faculty of Mathematics and Natural Sciences, University of Rzeszów, Pigonia 1, PL-35-310 Rzeszów, Poland.

Published by the American Physical Society under the terms of the Creative Commons Attribution 4.0 International license. Further distribution of this work must maintain attribution to the author(s) and the published article's title, journal citation, and DOI. Funded by SCOAP.
}

formulate equations for the scattering of photons off each other were formulated in 1925 [1]. Shortly thereafter, Louis de Broglie associated possible solutions of these equations to nontrivial scattering between two photons, in violation of the superposition principle [2]. This breakdown of the superposition principle can be incorporated in nonlinear Lagrangians, which result in nonlinear field equations. Attempts to solve the infinite Coulomb energy of a point source led to Born-Infeld electrodynamics, which effectively confirms the linearity down to some length scale, and introduces nonlinearities at smaller scales [3]. Virtual electron-positron pairs were suggested in 1933 to be at the origin of this photon-photon scattering [4]. The continued study of photon-photon scattering led to the Euler-Kockel-Heisenberg Lagrangian which modifies the classical Maxwell's equation in vacuum by leading nonlinear terms [5]. A more detailed account of the history of photon-photon scattering can be found in a recent review [6].

The advent of accelerating heavy-ions to ultrarelativistic energies with large associated photon fluxes provides new opportunities in gaining insight into the mechanisms of photon-photon scattering [7,8]. At these energies, not only lepton and quark loop diagrams constitute the signal, but also meson-exchange currents are predicted 
to contribute [9]. Such measurements in heavy-ion collisions hence extend photon-photon scattering from a pure QED issue to a subject connecting QED with QCD topics. This QED-QCD connection is, for example, an important ingredient in the quantum electrodynamical calculation of the muon anomalous moment (see e.g., [10,11] and references therein). In these calculations, light-by-light diagrams appear inside more complicated QCD-QED diagrams. There are proposals to study such diagrams in double back-Compton scattering using high-power lasers [12].

First evidence of diphoton measurements in ultraperipheral heavy-ion collisions have been reported by the ATLAS and CMS Collaborations [13,14]. These data are, however, restricted to photon-photon invariant masses $\mathrm{W}_{\gamma \gamma}>5$ and $6 \mathrm{GeV}$ for the CMS and ATLAS analysis, respectively. ATLAS measured a fiducial cross section of $\sigma=70 \pm 24$ (stat.) \pm 17 (syst.) $\mathrm{nb}$ and theoretical calculations (including experimental acceptance) gave $45 \pm 9 \mathrm{nb}$ [7] and $49 \pm$ $10 \mathrm{nb}$ [8]. ATLAS comparison of its experimental results to the predictions from Ref. [8] shows a reasonable agreement. Only 13 events were identified in the ATLAS data sample, with an expectation of 7.3 signal events and 2.6 background events from Monte Carlo simulations [13]. Recently, the CMS Collaboration measured the same process but for a slightly lower threshold of diphoton invariant mass [14]. The measured fiducial light-by-light scattering cross section, $\sigma=120 \pm 46$ (stat.) \pm 28 (syst.) \pm 4 (theo.) nb was obtained. The CMS measured value is in good agreement with the result obtained according to Ref. [15]. It is important to further test the light-by-light scatteringfor different energies and with a better precision.

The purpose of the study presented here is to examine the possibility of measuring photon-photon scattering in ultraperipheral heavy-ion collisions at LHC energies in the range $W_{\gamma \gamma}<5 \mathrm{GeV}$. At lower diphoton masses, photoproduction of meson resonances plays a role in addition to the Standard Model box diagrams [16], as well as double photon fluctuations into light vector mesons [8] or two-gluon exchanges [17]. In the present study, we restrict the invariant photon mass to $W_{\gamma \gamma}>0.4 \mathrm{GeV}$, and we include the photoproduction of the pseudoscalars $\eta, \eta^{\prime}(958)$. The assessment of the diphoton range $W_{\gamma \gamma}<0.4 \mathrm{GeV}$ is more elaborate since it includes photoproduction of the $\pi^{0}$, as well as feeddown from multi- $\pi^{0}$ decays of $\eta, \eta^{\prime}(958)$. The study of the diphoton range $W_{\gamma \gamma}<0.4 \mathrm{GeV}$ is beyond the scope of this paper, and will be the topic of a forthcoming study.

In the present study we consider also background from the $\gamma \gamma \rightarrow \pi^{0}(\rightarrow \gamma \gamma) \pi^{0}(\rightarrow \gamma \gamma)$ process measured e.g., by the Belle [18] and Crystal Ball [19] collaborations. In Ref. [9] a multicomponent model, which describes the Belle $\gamma \gamma \rightarrow$ $\pi^{0} \pi^{0}$ data, has been constructed. This model was used next to make predictions for the $A A \rightarrow A A \pi^{0} \pi^{0}$ reaction [9]. If only two photons from different neutral pions are measured within the experimental acceptance such an event could be wrongly identified as $\gamma \gamma \rightarrow \gamma \gamma$ scattering. Extra cuts need to be imposed to reduce or eliminate this background.

This paper is organized as follows. In Sec. II, the theoretical framework for calculating photon-photon scattering is outlined, the background from $\gamma \gamma \rightarrow \pi^{0} \pi^{0}$ is reviewed, and the cross section for photoproduction of resonances is studied. The experimental acceptances used for the given photon-photon scattering cross sections are described in Sec. III. The nuclear cross sections for lightby-light scattering in ultraperipheral lead-lead collisions at the energy $\sqrt{s_{N N}}=5.02 \mathrm{TeV}$ are given in Sec. IV. The treatment of the $\pi^{0} \pi^{0}$ background is discussed in Sec. V, and conclusions are presented in Sec. VI.

\section{THEORETICAL APPROACH FOR THE SIGNAL AND BACKGROUND}

We consider nuclear ultraperipheral collisions (UPCs). In Fig. 1 we illustrate the signal $(\gamma \gamma \rightarrow \gamma \gamma$ scattering) which we take to be the dominant box mechanism (see [8]). Panel (b) shows a diagram for $s$-channel $\gamma \gamma \rightarrow$ pseudoscalar/ scalar/tensor resonances which also contributes to the $\gamma \gamma \rightarrow$ $\gamma \gamma$ process. We also show [diagram (c)] the $\gamma \gamma \rightarrow \pi^{0} \pi^{0}$ process, which leads to what we consider as the dominant background when only one photon from each $\pi^{0} \rightarrow \gamma \gamma$ decay is detected. Other processes such as diffractive multi-hadron production that result in only two measured photons may also contribute to the background, but can be reduced using the techniques shown here. Being strongly dependent on the acceptance and detection thresholds, these processes need to be experimentally assessed and are beyond the scope of the current study.

Strong electromagnetic fields of fast moving nuclei are a source of photons. Due to coherent action of all protons in the (ultrarelativistic) nucleus, the wavelength of the photon is larger than the size of the nucleus. The coherence condition limits the photon virtuality to very low values given by the inverse of the nuclear radius squared according to the dependence: $Q^{2}=-q^{2}<\frac{1}{R^{2}} \approx(30 \mathrm{MeV})^{2}$. This value is much smaller than typical scales in the $\gamma \gamma$ fusion processes. Thus photons in ultrarelativistic heavy-ion collisions can be treated, to a very good approximation, as real photons. In the equivalent photon approximation (EPA) in impact parameter space, the phase space integrated cross section for $A_{1} A_{2} \rightarrow A_{1} A_{2} X_{1} X_{2}$ reaction is expressed through the five-fold integral,

$$
\begin{aligned}
& \sigma_{A_{1} A_{2} \rightarrow A_{1} A_{2} X_{1} X_{2}}\left(\sqrt{s_{A_{1} A_{2}}}\right) \\
& =\int \sigma_{\gamma \gamma \rightarrow X_{1} X_{2}}\left(W_{\gamma \gamma}\right) N\left(\omega_{1}, \mathbf{b}_{1}\right) N\left(\omega_{2}, \mathbf{b}_{2}\right) S_{a b s}^{2}(\mathbf{b}) \\
& \quad \times \mathrm{d}^{2} b \mathrm{~d} \bar{b}_{x} \mathrm{~d} \bar{b}_{y} \frac{W_{\gamma \gamma}}{2} \mathrm{~d} W_{\gamma \gamma} \mathrm{d} Y_{X_{1} X_{2}},
\end{aligned}
$$

where $X_{1} X_{2}$ is a pair of photons or neutral pions. $W_{\gamma \gamma}=$ $\sqrt{4 \omega_{1} \omega_{2}}$ and $Y_{X_{1} X_{2}}=\left(y_{X_{1}}+y_{X_{2}}\right) / 2$ are invariant mass and 


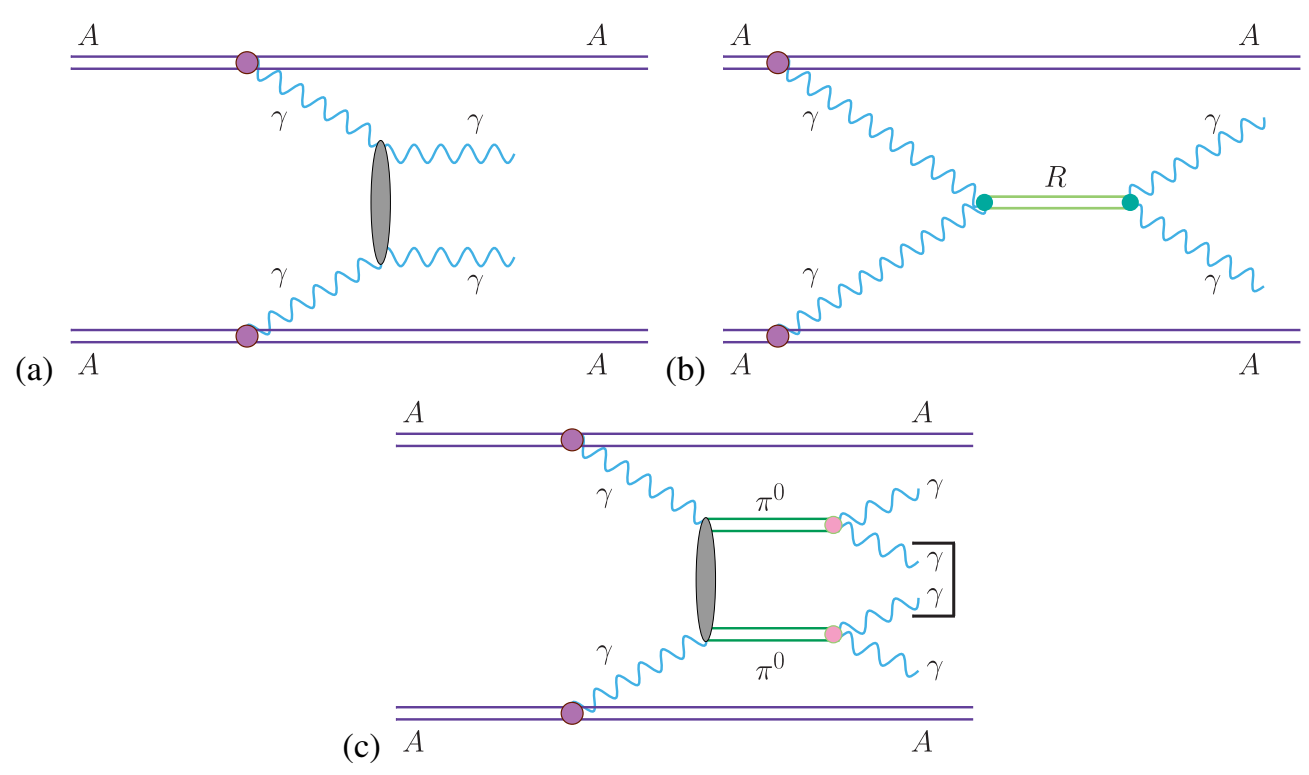

FIG. 1. The continuum $\gamma \gamma \rightarrow \gamma \gamma$ scattering (a), $\gamma \gamma \rightarrow$ resonances $\rightarrow \gamma \gamma$ (b), and the background mechanism (c).

rapidity of the outgoing $X_{1} X_{2}$ system. The energy of the photons is expressed through $\omega_{1 / 2}=W_{\gamma \gamma} / 2 \exp \left( \pm Y_{X_{1} X_{2}}\right)$, in the nucleus-nucleus center-of-mass frame. Experimental cuts on transverse momenta and rapidities are in the same frame, which for colliding beams is equivalent to the laboratory frame (the rest frame of the detectors). The variables $\mathbf{b}_{1}$ and $\mathbf{b}_{\mathbf{2}}$ are impact parameters of the photonphoton collision point with respect to parent nuclei 1 and 2 , respectively, and $\mathbf{b}=\mathbf{b}_{\mathbf{1}}-\mathbf{b}_{\mathbf{2}}$ is the standard impact parameter for the $A_{1} A_{2}$ collision. The absorption factor $S_{a b s}^{2}(\mathbf{b})$ assures UPC implying that the nuclei do not undergo nuclear breakup. The photon fluxes $\left(N\left(\omega_{i}, \mathbf{b}_{\mathbf{i}}\right)\right)$ are expressed through a nuclear charge form factor of the nucleus. In our calculations we use a realistic form factor which is a Fourier transform of the charge distribution in the nucleus. More details can be found e.g., in Ref. [20]. We expect that the equivalent photon approximation should be reliable for $W_{\gamma \gamma}>0.5 \mathrm{GeV}$ but its accuracy deserves future study.

The elementary cross section $\sigma_{\gamma \gamma \rightarrow X_{1} X_{2}}$ in Eq. (2.1) for the $\gamma \gamma \rightarrow \gamma \gamma$ scattering is calculated within LO QED with fermion loops (see left panel of Fig. 1 in Ref. [8]). The one-loop box diagrams were calculated with the help of the Mathematica package FORMCALC [21] and the LoOpToOLs library based on [22] to evaluate one-loop integrals. In the numerical calculations we include box diagrams with leptons and quarks only. At low energies, say $M_{\gamma \gamma}<5 \mathrm{GeV}$, there is no common agreement on the reliability of calculations with quarks/antiquarks. It is not clear which values of their masses should be used. Due to fractional charge of quarks their contribution is smaller than that of electrons or muons. However, all contributions add coherently and due to interference effect they may modify (enhance) the cross section by up to a factor of 2 . At low energies one could also include pionic loops. The relevant calculations are not trivial and require further study. One would need to include hadronic form factors since the charged pions in the loop may be off mass shell but in this case it is not clear how to ensure gauge invariance. Pionic loops were discussed recently in the context of hadronic light-by-light corrections to the anomalous magnetic moment of muon [23-26]. Inclusion of the $W$-boson loops is not necessary because this contribution is important only for energies larger than twice the $W$ boson mass [27]. Our results have been compared to and agree with Refs. [28-30]. Other production mechanisms were considered in Refs. [8,17], but their contributions are much smaller in the low diphoton mass region $M_{\gamma \gamma}<5 \mathrm{GeV}$ considered here.

\section{A. Pion pair production}

The elementary cross section for $\gamma \gamma \rightarrow \pi \pi$ was studied in detail in Ref. [9]. Both $\gamma \gamma \rightarrow \pi^{+} \pi^{-}$and $\gamma \gamma \rightarrow \pi^{0} \pi^{0}$ reactions were considered within the physical framework describing existing experimental data. Two of us calculated, for the first time, both the total cross section and angular distributions and demonstrated significance of resonances, continuum and $\mathrm{pQCD}$ mechanisms in these processes. Following [9], here we include nine resonances, $\gamma \gamma \rightarrow$ $\pi^{+} \pi^{-} \rightarrow \rho^{ \pm} \rightarrow \pi^{0} \pi^{0}$ continuum, Brodsky-Lepage and handbag mechanisms. A detailed formalism and description of these subprocesses can be found in Ref. [9]. The angular distribution for the $\gamma \gamma \rightarrow \pi^{0} \pi^{0}$ process can be written in standard form with the help of the $\lambda_{1}, \lambda_{2}$ photon helicity-dependent amplitudes, as a function of $z=\cos \theta$, where $\theta$ is the pion scattering angle 


$$
\begin{aligned}
\frac{\mathrm{d} \sigma_{\gamma \gamma \rightarrow \pi^{0} \pi^{0}}\left(W_{\gamma \gamma}\right)}{\mathrm{d} z}= & \frac{\sqrt{\frac{W_{\gamma \gamma}^{2}}{4}-m_{\pi}^{2}}}{\frac{W_{\gamma \gamma}}{2}} \frac{4 \pi}{4 \times 64 \pi^{2} W_{\gamma \gamma}^{2}} \\
& \times \sum_{\lambda_{1}, \lambda_{2}}\left|\mathcal{M}_{\gamma \gamma \rightarrow \pi^{0} \pi^{0}}\left(\lambda_{1}, \lambda_{2}\right)\right|^{2} .
\end{aligned}
$$

We use the formalism sketched above to calculate the multidimensional distribution

$$
\begin{aligned}
& \frac{\mathrm{d} \sigma_{A_{1} A_{2} \rightarrow A_{1} A_{2} \pi_{1}^{0} \pi_{2}^{0}}\left(\sqrt{s_{A_{1} A_{2}}}\right)}{\mathrm{d} y_{\pi_{1}^{0}} \mathrm{~d} y_{\pi_{2}^{0}} \mathrm{~d} p_{t, \pi^{0}}} \\
& =\int \frac{\mathrm{d} \sigma_{\gamma \gamma \rightarrow \pi_{1}^{0} \pi_{2}^{0}}\left(W_{\gamma \gamma}\right)}{\mathrm{d} z} N\left(\omega_{1}, \mathbf{b}_{1}\right) N\left(\omega_{2}, \mathbf{b}_{2}\right) S_{a b s}^{2}(\mathbf{b}) \\
& \quad \times \mathrm{d}^{2} b \mathrm{~d} \bar{b}_{x} \mathrm{~d} \bar{b}_{y} \frac{W_{\gamma \gamma}}{2} \frac{\mathrm{d} W_{\gamma \gamma} \mathrm{d} Y_{\pi_{1}^{0} \pi_{2}^{0}}}{\mathrm{~d} y_{\pi_{1}^{0}} \mathrm{~d} y_{\pi_{2}^{0}} \mathrm{~d} p_{t, \pi^{0}}} \mathrm{~d} z,
\end{aligned}
$$

for the $A_{1} A_{2} \rightarrow A_{1} A_{2} \pi^{0} \pi^{0}$ reaction. Here, $y_{\pi_{1}^{0}}, y_{\pi_{2}^{0}}$ are the rapidities of the first and second pion, $p_{t, \pi^{0}}$ is the transverse momentum of the pions (identical in our LO approximation) and $z$ is the pion center-of-mass scattering angle. Integration of this formula allows comparison with Eq. (2.1).

A dense three-dimensional grid of the triple differential cross section of Eq. (2.3) is prepared in a broad range of rapidities of both neutral pions and transverse momentum of one of them. A Monte Carlo code has been written to include radiative decays of both pions. As the pions are spin-0 mesons, the decays are taken to be isotropic in the rest frames of the decaying pions. Lorentz boosts are performed to obtain the kinematic distributions of photons in the laboratory (nucleus-nucleus center of mass) frame. Then logical conditions and cuts on the photons are imposed and distributions in different variables are obtained by an appropriate binning in the selected kinematic variable. The distributions are presented below and different experimental requirements are examined in order to estimate whether the $\gamma \gamma \rightarrow \gamma \gamma$ process can be observed.

\section{B. Resonance contributions}

The angular distribution for the $s$-channel resonances is typically used in calculating Feynman diagram contributions in the form:

$\frac{\mathrm{d} \sigma_{\gamma \gamma \rightarrow R \rightarrow \gamma \gamma}\left(W_{\gamma \gamma}\right)}{\mathrm{d} \cos \theta}=\frac{1}{32 \pi W_{\gamma \gamma}^{2}} \frac{1}{4} \sum_{\lambda_{1}, \lambda_{2}}\left|\mathcal{M}_{\gamma \gamma \rightarrow R \rightarrow \gamma \gamma}\left(\lambda_{1}, \lambda_{2}\right)\right|^{2}$,

where $\theta$ denotes the polar angle between the beam direction and the outgoing nucleon in the c.m. frame, $W_{\gamma \gamma}$ is the invariant mass of the $\gamma \gamma$ system. The amplitudes for the $\gamma \gamma$ production through the $s$-channel exchange of a pseudoscalar/scalar meson are written as

$$
\begin{aligned}
\mathcal{M}_{\gamma \gamma \rightarrow R \rightarrow \gamma \gamma}\left(\lambda_{1}, \lambda_{2}\right)= & \frac{\sqrt{64 \pi^{2} W_{\gamma \gamma}^{2} \Gamma_{R}^{2} \mathrm{Br}^{2}(R \rightarrow \gamma \gamma)}}{\hat{s}-m_{R}^{2}-i m_{R} \Gamma_{R}} \\
& \times \frac{1}{\sqrt{2 \pi}} \delta_{\lambda_{1}-\lambda_{2}} .
\end{aligned}
$$

Here we use the same notation as in Ref. [9]. In the present analysis we take into account only pseudoscalar and scalar mesons: $\eta, \eta^{\prime}(958), \eta_{c}(1 S), \eta_{c}(2 S), \chi_{c 0}(1 P)$. Their masses $m_{R}$, total widths $\Gamma_{R}$ and branching ratios $\operatorname{Br}(R \rightarrow \gamma \gamma)$ are taken from the PDG [31].

To calculate a resonance that decays into two photons, we use the following expression

$$
\begin{aligned}
& \frac{\mathrm{d} \sigma_{A_{1} A_{2} \rightarrow A_{1} A_{2} \gamma \gamma}\left(\sqrt{s_{A_{1} A_{2}}}\right)}{\mathrm{d} y_{\gamma_{1}} \mathrm{~d} y_{\gamma_{2}} \mathrm{~d} p_{t, \gamma}} \\
& =\int \frac{\mathrm{d} \sigma_{\gamma \gamma \rightarrow R \rightarrow \gamma \gamma}\left(W_{\gamma \gamma}\right)}{\mathrm{d} \cos \theta} N\left(\omega_{1}, \mathbf{b}_{1}\right) N\left(\omega_{2}, \mathbf{b}_{2}\right) S_{a b s}^{2}(\mathbf{b}) \\
& \quad \times \mathrm{d}^{2} b \mathrm{~d} \bar{b}_{x} \mathrm{~d} \bar{b}_{y} \frac{W_{\gamma \gamma}}{2} \frac{\mathrm{d} W_{\gamma \gamma} \mathrm{d} Y_{\gamma_{1} \gamma_{2}}}{\mathrm{~d} y_{\gamma_{1}} \mathrm{~d} y_{\gamma_{2}} \mathrm{~d} p_{t, \gamma}} \mathrm{d} \cos \theta
\end{aligned}
$$

for the calculation of the nuclear cross section. This formula includes the unpolarized differential elementary cross section of Eq. (2.4). Further discussions of the cross section for the mesonic resonant states in the context of light-by-light scattering are in Ref. [16].

In Ref. [16] a coherent sum of $s$-, $t$ - and $u$-contributions was included (see Fig. 2 therein). Inclusion of the $t$ - and $u$-channels leads to broad continua but they are several orders of magnitude smaller than the main $s$-channel contribution. The $t$ - and $u$-contributions are negligible close to the resonance position. They are also significantly smaller than the contribution of "standard" fermionic loops. We think, as will be discussed in this paper, that at least observation of the

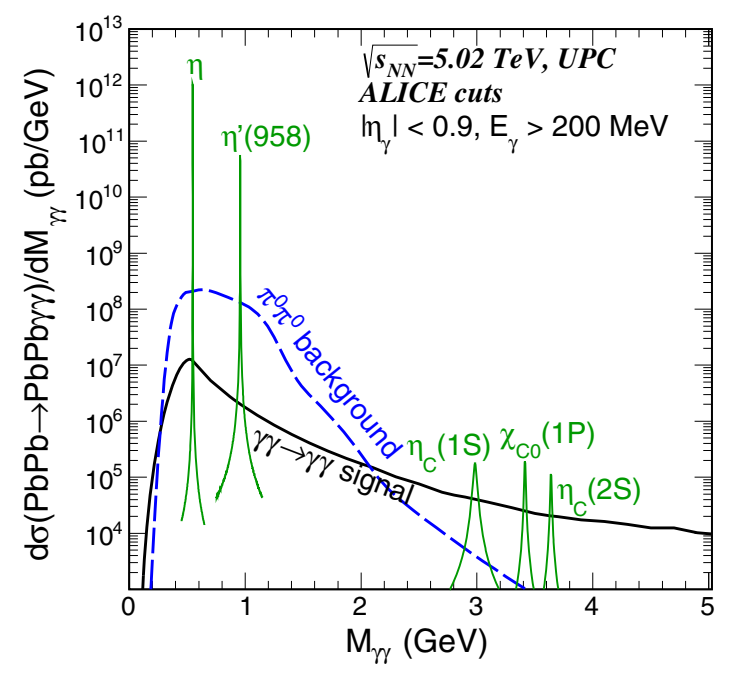

FIG. 2. Differential cross section as a function of invariant diphoton mass within the ALICE fiducial region. 
$\eta, \eta^{\prime}(958)$, etc., peaks will be possible at the LHC. Therefore in practice the contribution of $t$ - and $u$-channel resonance exchanges may be safely omitted in the present study. The $s$-channel resonant contribution seems to be sufficient in our analysis.

\section{EXPERIMENTAL ACCEPTANCE AND RESOLUTION}

We briefly summarize the experimental acceptance for measuring two-photon final states in Run 3 and beyond at the LHC, and describe the experimental resolution used in deriving our results. To illustrate our case, we take the acceptance of the ALICE central barrel at midrapidity, and the $\mathrm{LHCb}$ spectrometer acceptance at forward rapidities.

The ALICE central barrel covers the pseudorapidity range $|\eta|<0.9$ [32]. Within this range, ALICE is capable of measuring photons by different methods. First, electromagnetic calorimeters EMCal and PHOS cover part of the solid angle [33,34]. Second, photons can be detected by the photon conversion method (PCM). In this approach, photons which convert into $e^{+} e^{-}$pairs in the detector material are reconstructed by detecting the two charged tracks of the lepton pair. Whereas the photon measurement can be carried out with high efficiency but limited solid angle by the electromagnetic calorimeters, the measurement by PCM covers the full solid angle of the central barrel but suffers from reduced efficiency. Additionally, hybrid measurements are possible with one photon being detected by the calorimeters, and the other photon being reconstructed by the PCM. Analyses of $\pi^{0}$ and $\eta$ meson production in proton-proton collisions at $\sqrt{s}=8 \mathrm{TeV}$ by PCM in the ALICE central barrel resulted in a mass resolution $\sigma_{M}$ of the $\eta$ meson $\sigma_{M_{\eta}} \sim 5 \mathrm{MeV}$ (see Fig. 4 in Ref. [35]), from which a PCM energy resolution for single photons of $\sigma_{E_{\gamma}} / E_{\gamma} \sim 1.3 \%$ is deduced. For the azimuthal angular resolution $\sigma_{\phi}$, we choose a value of $\sigma_{\phi}=$ 0.02 in order to illustrate the different behavior of scalar and vector asymmetries explained and used below for suppressing the background. A comparative study of photon measurements by electromagnetic calorimeters to the PCM for measuring light-by-light scattering in the energy range considered, as well as a comprehensive analysis of the experimental resolutions, requires detailed studies of the response of the detectors used. Such an analysis is beyond the scope of this paper.

$\mathrm{LHCb}$ is fully instrumented in the pseudorapidity range $2<\eta<4.5$ with tracking, calorimetry and particle identification. Tracks can be reconstructed down to a transverse momentum of about $100 \mathrm{MeV}$, and photons down to a transverse energy of about $200 \mathrm{MeV}$. In this study we assume that any photon with $E_{t, \gamma}>200 \mathrm{MeV}$ and $2<\eta<$ 4.5 will be detected by LHCb, while photons outside this region are undetected. The energy resolution is parametrized as [36]:
TABLE I. Fiducial regions used in the present study.

\begin{tabular}{lcc}
\hline \hline Experiment & Pseudorapidity range & Energy cut \\
\hline ALICE & $-0.9<\eta_{\gamma}<0.9$ & $E_{\gamma}>0.2 \mathrm{GeV}$ \\
LHCb & $2.0<\eta_{\gamma}<4.5$ & $E_{t, \gamma}>0.2 \mathrm{GeV}$ \\
\hline \hline
\end{tabular}

$$
\frac{\sigma_{E_{\gamma}}}{E_{\gamma}}=\frac{0.085}{\sqrt{E_{\gamma}}}+\frac{0.003}{E_{\gamma}}+0.008,
$$

where $E_{\gamma}$ is the photon energy in GeV. These fiducial requirements and resolution allow us to make rough estimates for the feasibility of observing light-by-light scattering: a full study using the $\mathrm{LHCb}$ detector simulation would be necessary to obtain precise results (Ref. [37]).

The fiducial regions used in the present study are summarized in Table I. In the following they will be named "ALICE-fiducial" or "LHCb-fiducial" for brevity. It should be noted that for massless particles in the final state, $E_{t, \gamma}$ and $p_{t, \gamma}$, as well as rapidity and pseudorapidity are identical.

\section{CROSS SECTIONS}

We present the total cross section for different contributions to the diphoton final state in Table II. In this table, the total cross section is listed for two ranges of photon-photon invariant mass. The first range is from 0 to $2 \mathrm{GeV}$, with the second range for values $W_{\gamma \gamma}$ larger than $2 \mathrm{GeV}$. We take $W_{\gamma \gamma}^{\max }=50 \mathrm{GeV}$ for fermionic boxes, $W_{\gamma \gamma}^{\max }=5 \mathrm{GeV}$ for the $\pi^{0} \pi^{0}$ background (it is negligible above $W_{\gamma \gamma}=5 \mathrm{GeV}$ ) and $W_{\gamma \gamma} \in\left(m_{R}-1 \mathrm{GeV}, m_{R}+1 \mathrm{GeV}\right)$ for resonances. The $\pi^{0}$ resonance gives an important contribution in elastic photon-photon scattering at low energies. The corresponding cross section is fairly large. Due to special instrumentation at the LHC, even for the ALICE or LHCb experiments it is rather difficult to go to $W_{\gamma \gamma}<0.4 \mathrm{GeV}$. In addition, in this region the equivalent photon approximation [see Eq. (2.6)] may be not sufficiently accurate. Therefore in the following we do not include the $\pi^{0}$ resonance. Further feasibility studies are needed.

TABLE II. Total nuclear cross section in $\mathrm{nb}$ for the $\mathrm{Pb}-\mathrm{Pb}$ collision energy $\sqrt{s_{N N}}=5.02 \mathrm{TeV}$.

\begin{tabular}{|c|c|c|c|c|}
\hline \multirow{2}{*}{$\begin{array}{l}\text { Energy } \\
\text { Fiducial region }\end{array}$} & \multicolumn{2}{|c|}{$W_{\gamma \gamma}=(0-2) \mathrm{GeV}$} & \multicolumn{2}{|c|}{$W_{\gamma \gamma}>2 \mathrm{GeV}$} \\
\hline & ALICE & $\mathrm{LHCb}$ & ALICE & $\mathrm{LHCb}$ \\
\hline Boxes & 4890 & 3818 & 146 & 79 \\
\hline$\pi^{0} \pi^{0}$ background & 135300 & 40866 & 46 & 24 \\
\hline$\eta$ & 722573 & 568499 & & \\
\hline$\eta^{\prime}(958)$ & 54241 & 40482 & & \\
\hline$\eta_{c}(1 S)$ & & & 9 & 5 \\
\hline$\chi_{c 0}(1 P)$ & & & 4 & 2 \\
\hline$\eta_{c}(2 S)$ & & & 2 & 1 \\
\hline
\end{tabular}


The largest cross section is obtained for $\eta$ resonance production. The background contribution dominates over the signal up to $W_{\gamma \gamma}=2 \mathrm{GeV}$, however, as discussed in the following, can be reduced. Comparing results for $W_{\gamma \gamma}>2 \mathrm{GeV}$, the cross section for light-by-light scattering at midrapidity is about a factor 2 larger than at forward rapidity. The cross sections for both fermionic box and resonant signal are similar for the ALICE and LHCb fiducial regions.

\section{A. Differential cross section at midrapidity}

First we present some distributions within the ALICE fiducial region. The invariant mass distribution of two photon final states contains different contributions of signal and background as discussed in Sec. II. In Fig. 2, the contribution to the signal due to the Standard Model boxes is shown by the solid black line. The contributions to the signal by the different meson resonances are shown by the solid green lines, while the dashed blue line represents the $\pi^{0} \pi^{0}$ background as discussed in Sec. II A. The $\pi^{0} \pi^{0}$ background shown in Fig. 2 is composed of events where exactly two out of the four decay photons are within the fiducial volume, with the condition that each $\pi^{0}$ of the pair contributes one photon. At low invariant photon masses $W_{\gamma \gamma}<1.5 \mathrm{GeV}$, this background dominates over the signal by about an order of magnitude but can be reduced by taking into account the different phase space distribution of the signal and the background.

The two-dimensional distribution in rapidity of the first and second photon for signal and $\pi^{0} \pi^{0}$ background is shown in Fig. 3. The two distributions are peaked at forward and backward rapidities and are qualitatively rather similar, but differ by about two orders of magnitude. This figure shows that cuts on $\eta_{\text {sum }}=\eta_{1}+\eta_{2}$ could be used to reduce the background. However this leads to marginal

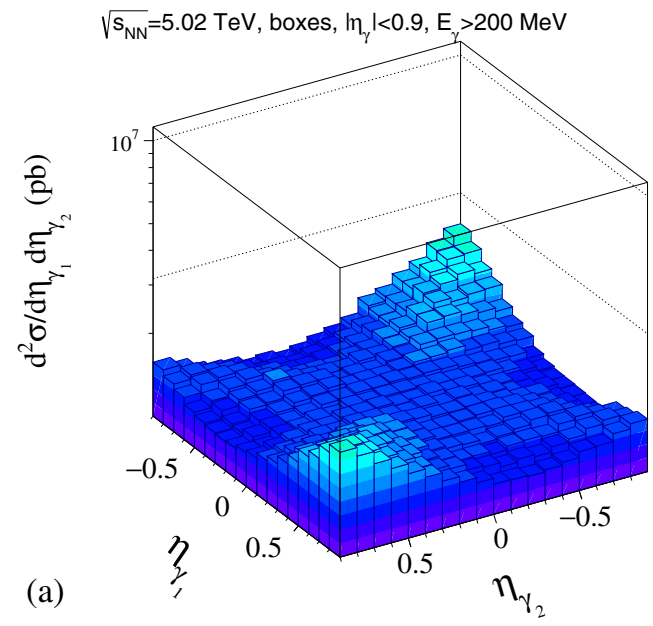

improvements due to the similarity in the shape of signal and background.

\section{B. Differential cross section at forward rapidity}

Equivalent distributions are now shown within the $\mathrm{LHCb}$ fiducial region. In Fig. 4 we show the diphoton invariant mass distribution. The distributions are similar to the ALICE conditions both in normalization and shape. The solid black line is the signal corresponding to the Standard Model box contribution, the solid green lines correspond to resonant mesonic states while the dashed line corresponds to the $\pi^{0} \pi^{0}$ background defined in the main text. The relative background is slightly lower than for ALICE but the conclusion again is that the signal can be clearly observed only for $W_{\gamma \gamma}>2 \mathrm{GeV}$ and one can observe very clear contributions coming from $\eta$ and $\eta^{\prime}(958)$ resonances. The inclusion of the LHCb energy resolution [Eq. (3.1)] broadens the peak in the distribution, which is plotted in Fig. 4 with and without energy smearing.

In Fig. 5 we show two-dimensional distributions as a function of pseudorapidity of the first and second photon. The left panel corresponds to the $\gamma \gamma \rightarrow \gamma \gamma$ (box) signal and the right panel shows the result for the $\pi^{0} \pi^{0}$ background. In some regions of $\left(\eta_{1} \times \eta_{2}\right)$ space the background contribution is much larger than the signal one. Note the different scale on $\mathrm{d}^{2} \sigma / \mathrm{d} \eta_{\gamma_{1}} \mathrm{~d} \eta_{\gamma_{2}}$-axis in the left and right panels. As in the case of the ALICE fiducial region (Fig. 3), the signal is two orders of magnitude smaller than the background but in the LHCb case the shapes of the distributions are rather different. Here there is relatively more background when both photons have large rapidities.

\section{BACKGROUND SUPPRESSION OF $\pi^{0} \pi^{0}$ DECAYS}

The background from the $\pi^{0} \pi^{0}$ decays shown in Fig. 2 can be reduced by kinematic cuts. Not taking into account

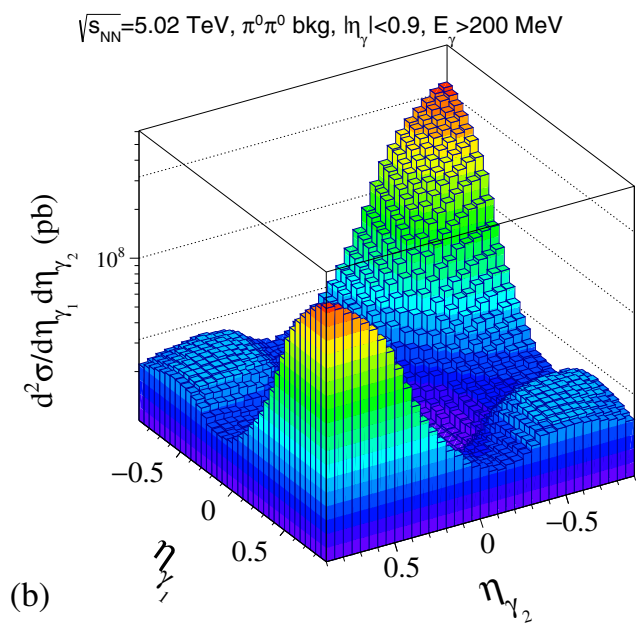

FIG. 3. Two-dimensional distribution of the signal (left panel) and $\pi^{0} \pi^{0}$ background (right panel) in rapidity of first and second photon (randomly chosen). 

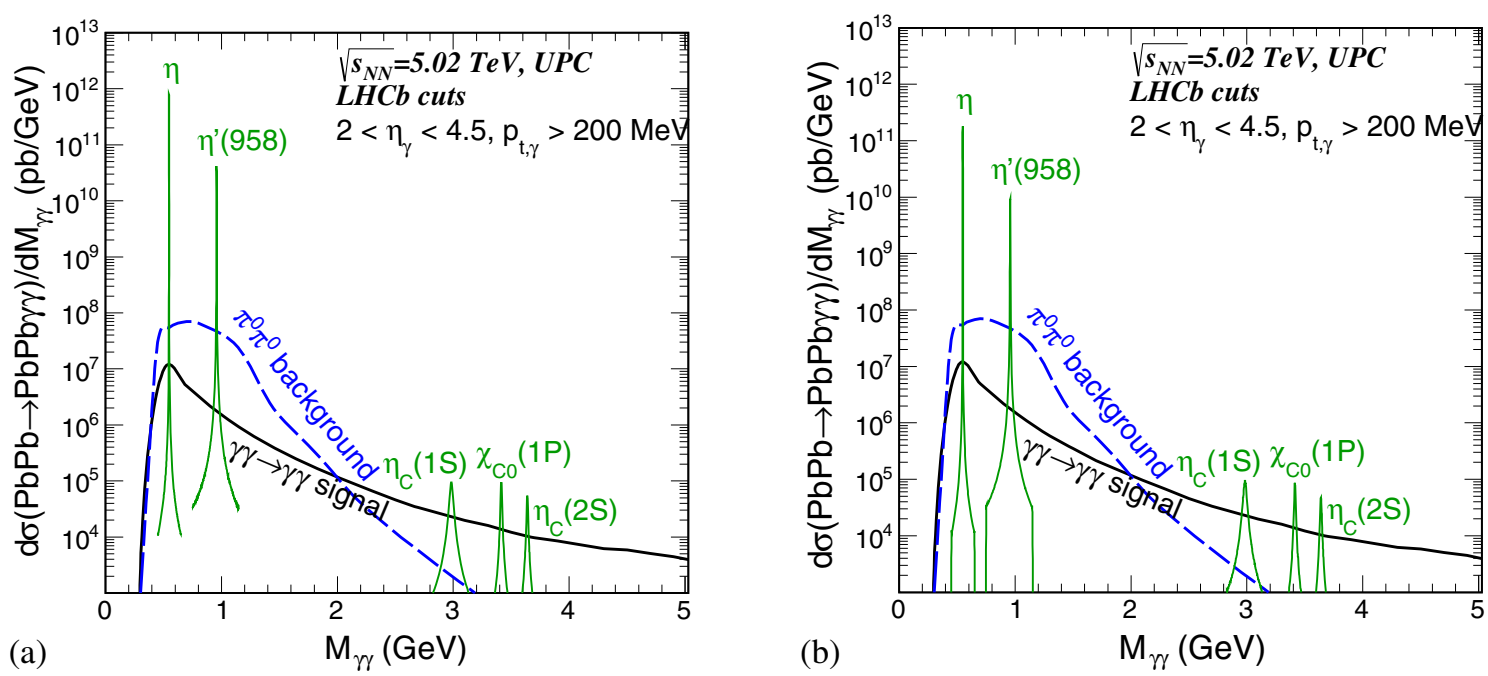

FIG. 4. Invariant diphoton mass distribution for the standard LHCb fiducial region presented without (a) and with (b) energy resolution.
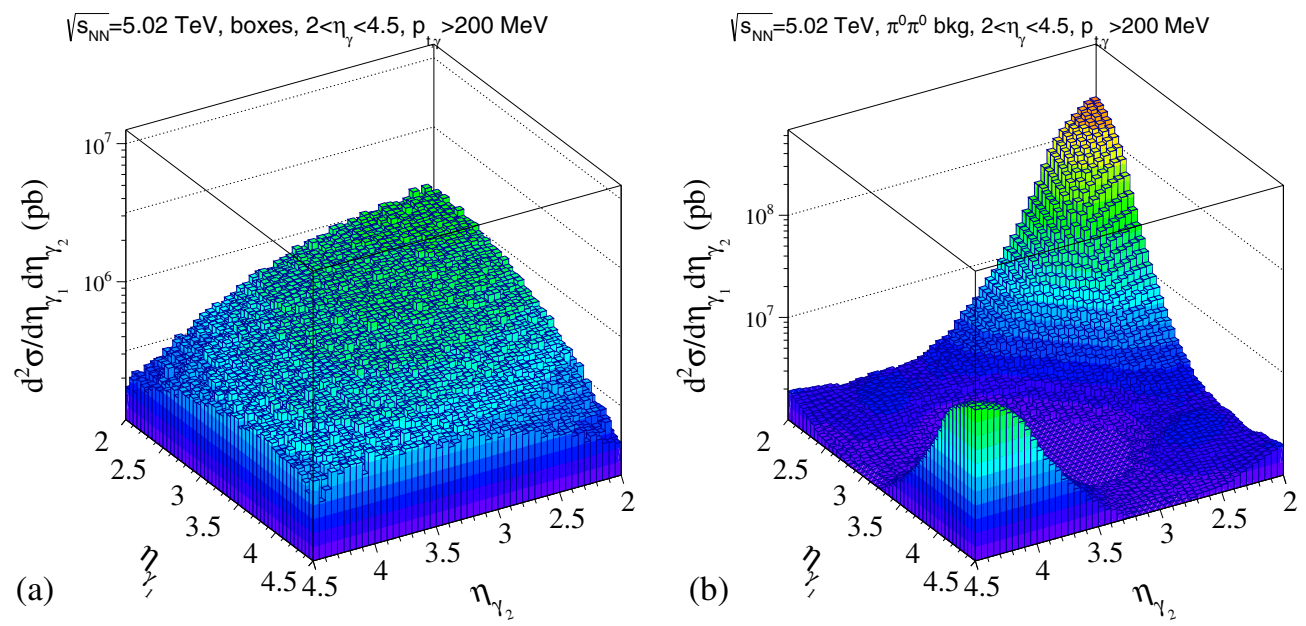

FIG. 5. Two-dimensional distribution of the signal (left panel) and background (right panel) in rapidity of first and second registered photon (chosen randomly).

the experimental resolution, the two final state photons of $\gamma \gamma \rightarrow \gamma \gamma$ scattering, shown in Fig. 1(a,b), are of equal transverse momenta and are back-to-back in azimuthal angle. In first order, the correlation in transverse momentum will be smeared out by the experimental resolution in the measurement of the two photons. Higher order corrections, such as the finite values of the beam emittance and the crossing angle of the colliding beams, are beyond the scope of the study presented here, and are hence neglected in the results presented below. The correlations of the signal can be quantified by two asymmetries,

$$
A_{S}=\left|\frac{\left|\vec{p}_{T}(1)\right|-\left|\vec{p}_{T}(2)\right|}{\left|\vec{p}_{T}(1)\right|+\left|\vec{p}_{T}(2)\right|}\right|
$$

$$
A_{V}=\frac{\left|\vec{p}_{T}(1)-\vec{p}_{T}(2)\right|}{\left|\vec{p}_{T}(1)+\vec{p}_{T}(2)\right|}
$$

Here, the scalar asymmetry $A_{S}$ is a measure of the relative difference in transverse momentum of the two photons, and is nonzero for two back-to-back photons of the signal due to the finite energy resolution of the measurement. The vector asymmetry $A_{V}$ defined in Eq. (5.2) reflects a convolution of the experimental resolutions of photon energy and azimuthal angle measurement. The two-photon background resulting from the $\pi^{0} \pi^{0}$ decays does not show the correlations discussed above. The correlations can hence be used to suppress the background by kinematical cuts on these two asymmetries. 

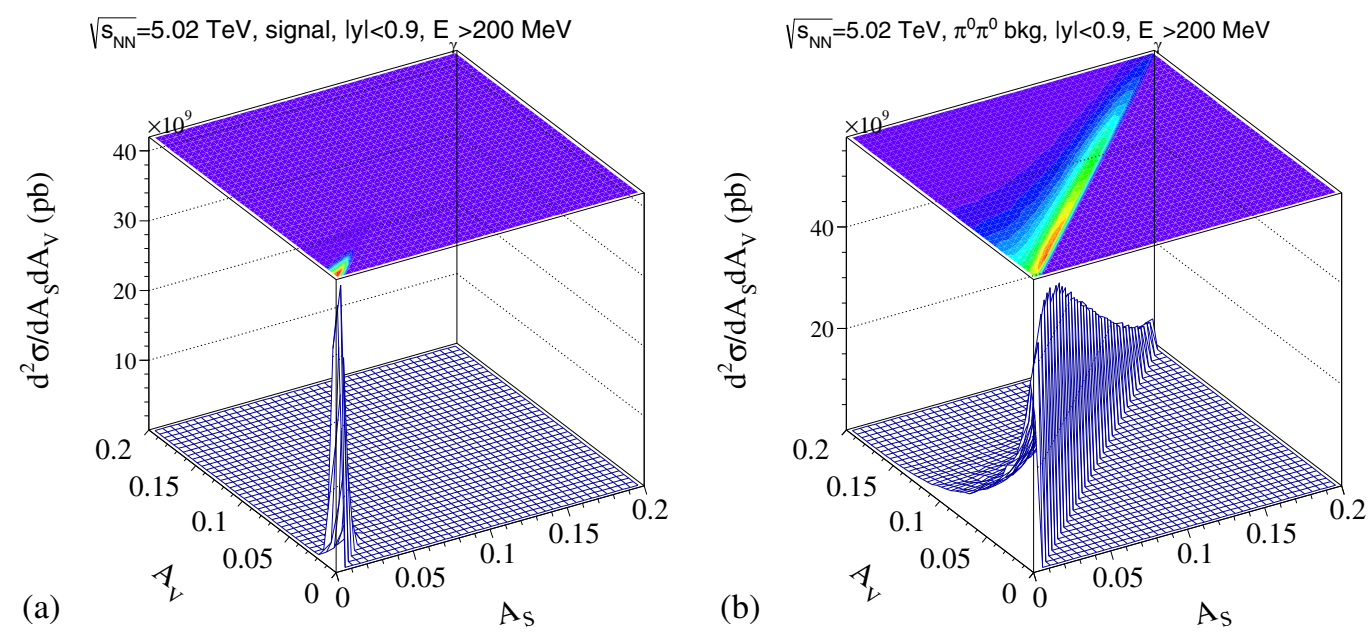

FIG. 6. Vector vs scalar asymmetry of signal (left panel) and background (right panel).

The correlation of the two asymmetries discussed above are shown for the signal in Fig. 6(a) for an energy resolution of $\sigma_{E_{\gamma}} / E_{\gamma}=1.3 \%$ and an azimuthal angle resolution of $\sigma_{\phi}=0.02$. From the considerations on asymmetries outlined above, one expects the vector asymmetry to be larger than the scalar asymmetry, $A_{V}>A_{S}$, as shown in Fig. 6(a). The corresponding histogram [Fig. 6(b)] is shown for photon background pairs resulting from the $\pi^{0} \pi^{0}$ decays. This background distribution is an order of magnitude wider compared to the distribution of the signal. A carefully chosen cut on the asymmetry parameters $A_{S}$ and $A_{V}$ will therefore reduce the background substantially, while reducing the signal only marginally.

The diphoton mass distribution is shown in Fig. 7 from a study performed within the ALICE fiducial region, with successive cuts on $A_{S}$ applied. The signal is reduced by a negligible amount for both values of $A_{S}$. The cut $A_{S}<0.02$ reduces the background by about a factor of 10 as shown by

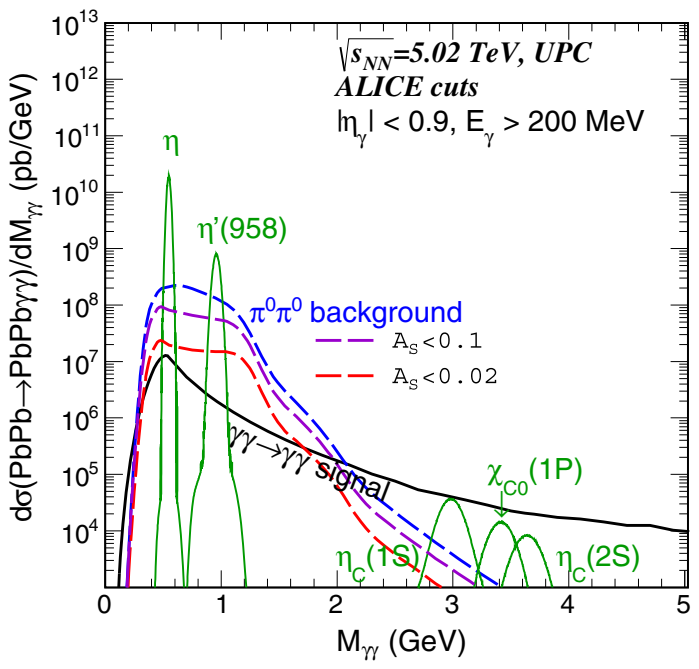

FIG. 7. Signal and background with asymmetry conditions. the red line, and results in a remaining background which is a factor of about 10 larger than the signal at diphoton masses $M_{\gamma \gamma} \sim 1.2 \mathrm{GeV}$.

A sideband subtraction method using the asymmetry parameter $A_{S}$ as separation variable can be used to extract the signal in this mass range. In this approach, kinematic distributions of signal and background, which can be one or multidimensional, are fit in intervals of the separation variable. For the diphoton signal a one-dimensional analysis could be based on the mass distribution while a higherdimensional approach could include the pseudorapidity distributions of the two photons shown in Fig. 3, and the transverse momentum distribution of the photon pair. To illustrate the one-dimensional approach, we show in Fig. 8 (a) the distribution of signal and background for the first three intervals of the separation variable, labeled signal region, sideband 1 and sideband 2 . The signal region, defined by $0<A_{S}<0.02$, contains $\sim 95 \%$ of the signal events. Sideband 1 and sideband 2 are defined by $0.02<$ $A_{S}<0.04$ and $0.04<A_{S}<0.06$, respectively. The distribution of the signal is at the $5 \%$ level in sideband 1 , and negligibly small in sideband 2, whereas the background distribution extends well into the sideband regions as shown in Fig. 8(a) by the dashed blue line. In Fig. 8(b), the mass distributions of background events in the signal and the two sideband regions are shown by the red-dashed, green-dotted and black-dot-dashed lines, respectively. Clearly visible in this panel is a continuous reduction of the background cross section from the signal to the sideband 2 region, as expected by the behavior of the background scalar asymmetry shown in Fig. 8(a). Based on the kinematical fits of the signal and background distributions, the sideband analysis derives a factor expressing the likelihood of the event belonging to the signal or background sample (see e.g., [38]). Quantitative results on the misidentification of background as signal events depend, however, on the event statistics available and information 

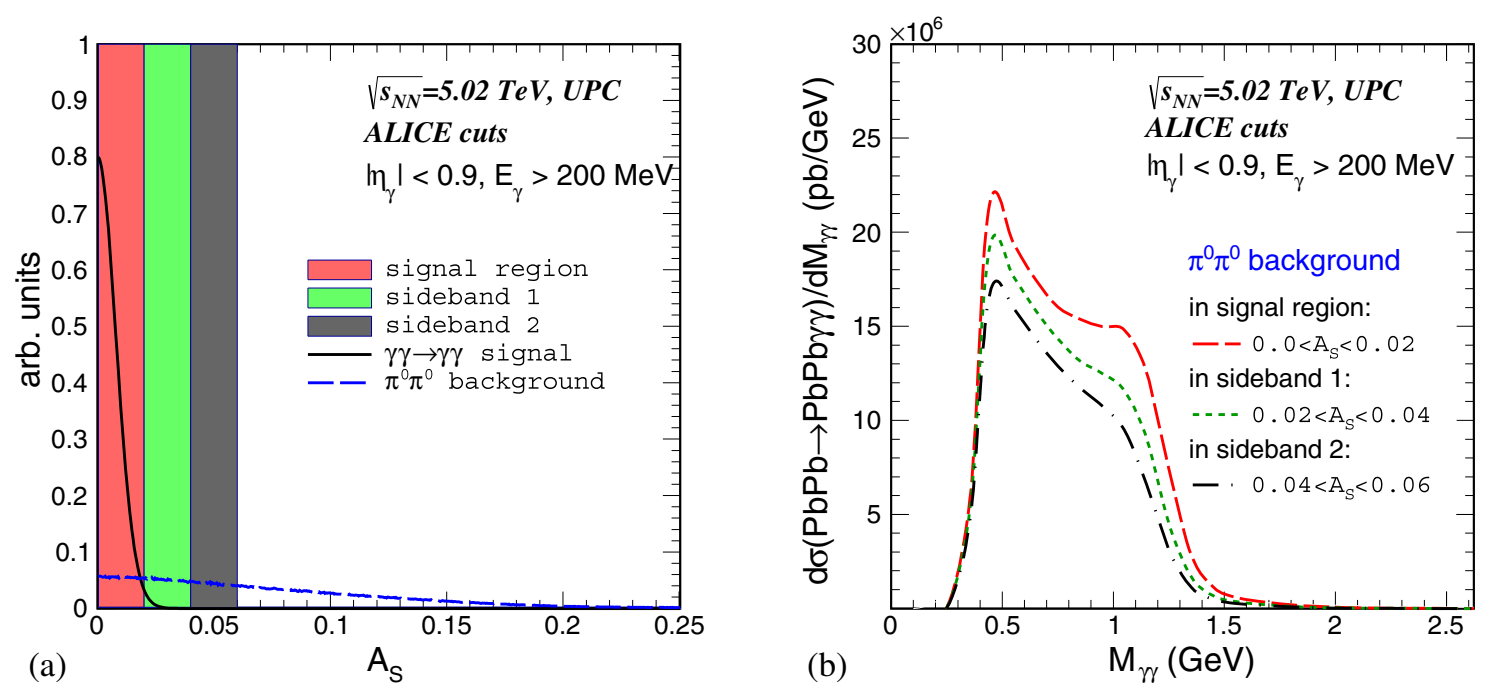

FIG. 8. Scalar asymmetry distribution of signal and background (a), and signal and sideband background mass distribution (b).

on the single photon detection efficiency, and are beyond the scope of the study presented here.

\section{CONCLUSIONS}

Ultraperipheral heavy-ion collisions at high energies open the possibility to measure $\gamma \gamma \rightarrow \gamma \gamma$ scattering. So far the ATLAS and CMS collaborations obtained first evidence of photon-photon scattering for invariant masses $W_{\gamma \gamma}>6$ and $5 \mathrm{GeV}$, respectively. Due to the experimental cuts on transverse photon momenta $p_{t, \gamma}>3 \mathrm{GeV}$, the resulting statistics is so far rather limited. The ATLAS result is roughly consistent with the Standard Model predictions for elementary cross section embedded into state-of-art nuclear calculation including realistic photon fluxes as the Fourier transform of realistic charge distribution.

Here we consider the possibility to study elastic $\gamma \gamma \rightarrow \gamma \gamma$ scattering in the diphoton mass range $W_{\gamma \gamma}<5 \mathrm{GeV}$ at the LHC using ALICE and LHCb detectors. Our results show that the contributions of the pseudoscalar resonances $\eta, \eta^{\prime}(958)$ are clearly visible on top of the diphoton mass continuum arising from lepton loop diagrams. We have made first predictions for cross sections as a function of diphoton mass for typical acceptances of the ALICE and $\mathrm{LHCb}$ experiments. The evaluation of counting rates needs, however, Monte Carlo simulations which take into account detailed acceptances and realistic responses of the detectors used for measuring two-photon final states.

In addition to the signal $(\mathrm{PbPb} \rightarrow \mathrm{PbPb \gamma \gamma})$ we consider the background dominated by the $P b P b \rightarrow P b P b \pi^{0} \pi^{0}$ reaction when only two out of the four decay photons in the final state are registered. This background can be reduced by imposing cuts on scalar and vector asymmetry of transverse momenta of the two photons. Cuts on sum of photon rapidities (or the rapidity of the diphoton system) can additionally be used to reduce the background. The background remaining after these cuts dominates the signal by a factor of about ten at diphoton masses $M_{\gamma \gamma} \sim 1.2 \mathrm{GeV}$. The extraction of the signal in this mass range is feasible by a multivariate sideband subtraction analysis. Quantitative results on the signal efficiency and background suppression in this sideband subtraction approach depend on the statistics of the data sample available for analysis.

In our study we take the dominant background shown in Fig. 1(c) as arising from the decay of two $\pi^{0}$ 's which are correlated by the emission from two vertices which, however, cannot be resolved at the macroscopic level due to detector resolution limitation. In a crossing of heavy-ion bunches, single $\pi^{0}$ production can occur by different pairs of particles in the two beams, resulting in multiple uncorrelated $\pi^{0}$ production. These $\pi^{0}$ 's emerge from different vertices which are spread along the interaction region of the two colliding beams. The contribution of the decay photons of these uncorrelated $\pi^{0}$ 's to the background depends on the beam parameters at the interaction point and the position resolution of the photon reconstruction along the beam direction, and is beyond the scope of the study presented here.

\section{ACKNOWLEDGMENTS}

The authors thank the ExtreMe Matter Institute EMMI at GSI, Darmstadt, for the support of the workshop "Challenges in Photon Induced Interactions" in Krakow where this study was initiated. This work has been supported by the Polish National Science Center Grant No. DEC-2014/15/B/ST2/02528 (M. K. G. and A. S.) and by the German Federal Ministry of Education and Research under promotional Reference No. 05P19VHCA1 (R. S.). 
[1] K. Schaposchnikow, Über Zusammenstöße von Lichtquanten, Z. Phys. 33, 706 (1925).

[2] L. de Broglie, Ondes et Mouvements, Collection de Physique Mathématique, Vol. 1 (Gauthier-Villars et Cie fditeurs, Paris, 1926).

[3] M. Born, Modified field equations with a finite radius of the electron, Nature (London) 132, 282 (1933).

[4] O. Halpern, Scattering processes produced by electrons in negative energy states, Phys. Rev. 44, 855 (1933).

[5] W. Heisenberg and H. Euler, Consequences of Dirac's theory of positrons, Z. Phys. 98, 714 (1936).

[6] K. Scharnhorst, Photon-photon scattering and related phenomena. Experimental and theoretical approaches: The early period, arXiv:1711.05194.

[7] D. d'Enterria and G. G. da Silveira, Observing Light-byLight Scattering at the Large Hadron Collider, Phys. Rev. Lett. 111, 080405 (2013); Erratum, Phys. Rev. Lett. 116, 129901(E) (2016).

[8] M. Kłusek-Gawenda, P. Lebiedowicz, and A. Szczurek, Light-by-light scattering in ultraperipheral $\mathrm{Pb}-\mathrm{Pb}$ collisions at energies available at the CERN Large Hadron Collider, Phys. Rev. C 93, 044907 (2016).

[9] M. Kłusek-Gawenda and A. Szczurek, $\pi^{+} \pi^{-}$and $\pi^{0} \pi^{0}$ pair production in photon-photon and in ultraperipheral ultrarelativistic heavy ion collisions, Phys. Rev. C 87, 054908 (2013).

[10] G. W. Bennett et al. (Muon g-2 Collaboration), Final report of the Muon E821 anomalous magnetic moment measurement at BNL, Phys. Rev. D 73, 072003 (2006).

[11] G. Colangelo, M. Hoferichter, M. Procura, and P. Stoffer, Hadronic light-by-light contribution to $(g-2)_{\mu}$ : A dispersive approach, EPJ Web Conf. 175, 01025 (2018).

[12] C. Bula et al. (E144 Collaboration), Observation of Nonlinear Effects in Compton Scattering, Phys. Rev. Lett. 76, 3116 (1996).

[13] M. Aaboud et al. (ATLAS Collaboration), Evidence for light-by-light scattering in heavy-ion collisions with the ATLAS detector at the LHC, Nat. Phys. 13, 852 (2017).

[14] A. M. Sirunyan et al. (CMS Collaboration), Evidence for light-by-light scattering and searches for axion-like particles in ultraperipheral $\mathrm{PbPb}$ collisions at $\sqrt{s_{\mathrm{NN}}}=5.02 \mathrm{TeV}$, arXiv:1810.04602 [Phys. Lett. B (to be published)].

[15] M. Kłusek-Gawenda, Importance of mesons in light-bylight scattering in ultraperipheral lead-lead collisions at the LHC, EPJ Web Conf. 199, 05004 (2019).

[16] P. Lebiedowicz and A. Szczurek, The role of meson exchanges in light-by-light scattering, Phys. Lett. B 772, 330 (2017).

[17] M. Kłusek-Gawenda, W. Schäfer, and A. Szczurek, Twogluon exchange contribution to elastic $\gamma \gamma \rightarrow \gamma \gamma$ scattering and production of two-photons in ultraperipheral ultrarelativistic heavy ion and proton-proton collisions, Phys. Lett. B 761, 399 (2016).

[18] S. Uehara et al. (Belle Collaboration), High-statistics study of neutral-pion pair production in two-photon collisions, Phys. Rev. D 79, 052009 (2009).

[19] H. Marsiske et al. (Crystal Ball Collaboration), A measurement of $\pi^{0} \pi^{0}$ production in two photon collisions, Phys. Rev. D 41, 3324 (1990).
[20] M. Kłusek-Gawenda and A. Szczurek, Exclusive muon-pair productions in ultrarelativistic heavy-ion collisionsrealistic nucleus charge form factor and differential distributions, Phys. Rev. C 82, 014904 (2010).

[21] T. Hahn and M. Perez-Victoria, Automatized one loop calculations in four-dimensions and D-dimensions, Comput. Phys. Commun. 118, 153 (1999).

[22] G. J. van Oldenborgh and J.A. M. Vermaseren, New algorithms for one loop integrals, Z. Phys. C 46, 425 (1990).

[23] G. Colangelo, M. Hoferichter, M. Procura, and P. Stoffer, Dispersion relation for hadronic light-by-light scattering: Two-pion contributions, J. High Energy Phys. 04 (2017) 161.

[24] G. Colangelo, M. Hoferichter, M. Procura, and P. Stoffer, Rescattering Effects in the Hadronic-Light-by-Light Contribution to the Anomalous Magnetic Moment of the Muon, Phys. Rev. Lett. 118, 232001 (2017).

[25] I. Danilkin and M. Vanderhaeghen, Light-by-light scattering sum rules in light of new data, Phys. Rev. D 95, 014019 (2017).

[26] I. Danilkin, C. F. Redmer, and M. Vanderhaeghen, The hadronic light-by-light contribution to the muon's anomalous magnetic moment, arXiv:1901.10346.

[27] P. Lebiedowicz, R. Pasechnik, and A. Szczurek, Search for technipions in exclusive production of diphotons with large invariant masses at the LHC, Nucl. Phys. B881, 288 (2014).

[28] G. Jikia and A. Tkabladze, Photon-photon scattering at the photon linear collider, Phys. Lett. B 323, 453 (1994).

[29] Z. Bern, A. De Freitas, L. J. Dixon, A. Ghinculov, and H. L. Wong, QCD and QED corrections to light by light scattering, J. High Energy Phys. 11 (2001) 031.

[30] D. Bardin, L. Kalinovskaya, and E. Uglov, Standard Model light-by-light scattering in SANC: Analytic and numeric evaluation, Phys. At. Nucl. 73, 1878 (2010).

[31] C. Patrignani et al. (Particle Data Group), Review of particle physics, Chin. Phys. C 40, 100001 (2016).

[32] B. Abelev et al. (ALICE Collaboration), Performance of the ALICE experiment at the CERN LHC, Int. J. Mod. Phys. A 29, 1430044 (2014).

[33] J. Allen et al. (ALICE EMCal Collaboration), Performance of prototypes for the ALICE electromagnetic calorimeter, Nucl. Instrum. Methods Phys. Res., Sect. A 615, 6 (2010).

[34] B. Abelev et al. (ALICE Collaboration), Neutral pion production at midrapidity in $\mathrm{pp}$ and $\mathrm{Pb}-\mathrm{Pb}$ collisions at $\sqrt{s_{N N}}=2.76 \mathrm{TeV}$, Eur. Phys. J. C 74, 3108 (2014).

[35] S. Acharya et al. (ALICE Collaboration), $\pi^{0}$ and $\eta$ meson production in proton-proton collisions at $\sqrt{s}=8 \mathrm{TeV}$, Eur. Phys. J. C 78, 263 (2018).

[36] C. Abellan Beteta et al., Time alignment of the front end electronics of the LHCb calorimeters, J. Instrum. 7, P08020 (2012).

[37] M. Clemencic, G. Corti, S. Easo, C. R. Jones, S. Miglioranzi, M. Pappagallo, and P. Robbe (LHCb Collaboration), The LHCb simulation application, Gauss: Design, evolution and experience, J. Phys. Conf. Ser. 331, 032023 (2011).

[38] M. Williams, M. Bellis, and C. A. Meyer, Multivariate side-band subtraction using probabilistic event weights, J. Instrum. 4, P10003 (2009). 